\title{
A Randomized Clinical Trial of a Care Recommendation Letter Intervention for Somatization in Primary Care
}

\author{
W. Perry Dickinson, $M D^{1}$ \\ L. Miriam Dickinson, $P b D^{1}$ \\ Frank $V$. deGruy, MD, MSFM ${ }^{1}$ \\ Deborab S. Main, $P b D^{1}$ \\ Lucy M. Candib, $M D^{2}$ \\ Katbryn Rost, $P b D^{1}$
}

'Department of Family Medicine, University of Colorado Health Sciences Center, Denver, Colo

${ }^{2}$ Family Health Center of Worcester and Department of Family Medicine and Community Health, University of Massachusetts Medical School, Worcester, Mass

Conflicts of interest: None reported

\section{CORRESPONDING AUTHOR}

Perry Dickinson, MD

University of Colorado Health Sciences Center

Department of Family Medicine

PO Box 6508

12474 E 19th Ave, Building 402

Aurora, CO 80045-0508

perry.dickinson@uchsc.edu

\begin{abstract}
PURPOSE This paper describes the impact of a care recommendation (CR) letter intervention on patients with multisomatoform disorder (MSD) and analysis of patient factors that affect the response to the intervention.

METHODS One hundred eighty-eight patients from 3 family practices, identified through screening of 2,902 consecutive patients, were classified using somatization diagnoses based on the number of unexplained physical symptoms from a standardized mental health interview. In a controlled, single-crossover trial, patients were randomized to have their primary care physician receive the CR letter either immediately following enrollment or 12 months after enrollment. The CR letter notified the physician of the patient's somatization status and provided recommendations for the patient's care. Patients were followed for 24 months with assessments of functional status at baseline, 12, and 24 months.
\end{abstract}

RESULTS Longitudinal analysis revealed a 12-month intervention effect for patients with multisomatoform disorder (MSD) of 5.5 points $(P<.001)$ on the physical functioning (PCS) scale of the SF-36. Analysis of scores on the MCS scale of the SF-36 found no significant effect on mental functioning. The intervention was more effective for patients with 1 or more comorbid chronic physical diseases $(P=.01)$.

CONCLUSIONS The CR letter has a favorable impact on physical impairment of primary care patients with MSD, especially for patients with comorbid chronic physical disease. Multisomatoform disorder appears to be a useful diagnostic classification for managing and studying somatization in primary care patients.

Ann Fam Med 2003;1:228-235. DOI: 10.1370/afm.5.

\section{INTRODUCTION}

$\mathrm{S}$ omatization is extremely common in primary care, with up to half of all primary care visits involving unexplained somatic complaints. ${ }^{1-4}$ Somatization is associated with considerable patient impairment and increased health care costs. ${ }^{5,6}$ The care of somatizing patients can also be frustrating for clinicians, at least in part because treatment options are limited. ${ }^{7-9}$

Various diagnostic classifications have been used for somatization, but in recent years 3 diagnoses have been most prominent. Somatization disorder (SD) is a severe form of somatization, defined by the DSM-III-R requirements of 13 of a list of 35 lifetime unexplained physical symptoms. ${ }^{10}$ To include patients with significant somatization who did not meet the threshold for SD, Escobar proposed and validated a less severe form of somatization called abridged somatization disorder (ASD), requiring at least 6 lifetime unexplained symptoms in women and 4 in men. ${ }^{11,12}$ Multisomatoform disorder (MSD) is a more recent diagnostic formulation based on current unexplained physical symptoms. ${ }^{13,14}$ Multisomatoform disorder 
has received increased attention because studies have documented instability of patient recall of lifetime somatization symptoms, upon which SD and ASD are based. ${ }^{15}$ Multisomatoform disorder requires the presence of 3 or more current (within the past 2 weeks) unexplained symptoms out of a list of 15 , along with a 2-year history of somatization. Since it depends on current symptoms, the diagnosis of MSD may have greater clinical utility and reliability than diagnoses based on recall of symptoms that may have long since disappeared. Multisomatoform disorder is much more common in primary care settings than somatization disorder, but with comparable levels of impairment. ${ }^{13,14}$

Even though somatization is common, debilitating, and expensive, researchers have directed relatively little attention to developing effective interventions for primary care. Pharmacological treatment with tricyclic or SSRI antidepressant medications, ${ }^{16,17}$ group therapy, ${ }^{18}$ cognitive behavioral therapy, ${ }_{1}^{19}$ and massage therapy ${ }^{20-22}$ have been shown to be beneficial for some patients.

One of the most-studied interventions for somatization has been a care recommendation (CR) letter sent to somatizing patients' primary care physicians. The letter identifies specific patients as having the somatization diagnosis and makes recommendations about appropriate care. ${ }^{18,23-25}$ This intervention has been shown to decrease costs associated with the care of the somatizing patients and to lead to no deterioration or to improved physical functioning for those patients. The CR letter intervention's effect seems quite robust, with comparable impacts in patients with somatization disorder ${ }^{18,23,24}$ and subthreshold somatization. ${ }^{25}$ The CR letter is very promising and may offer an inexpensive and effective first-stage intervention for somatization. Previous studies of the CR letter have been conducted in populations referred for intervention by their primary care physicians, and these patients may have more severe forms of somatization than are normally found in primary care. The studies do not tell us how well the intervention would work with representative samples of somatizing primary care patients.

As part of the Somatization in Primary Care Study, we sought to assess the effects of the CR letter intervention on physical and emotional functioning for a representative sample of primary care patients with MSD. Our primary hypothesis was that patients with MSD whose physicians received the CR letter would demonstrate significantly better physical and emotional functioning after 1 year than patients receiving usual care. To address the comparability of the CR letter's effects in MSD to previous research, we also assessed the intervention's effect on patients who met diagnostic criteria for ASD and SD. Our secondary goal was to determine if there were patient factors such as comor- bid chronic physical or current psychiatric disease or demographic factors that had an impact on the effectiveness of the intervention in MSD patients.

\section{METHODS}

The sample for this study consists of 188 patients who met criteria for 1 or more somatization diagnoses from the Somatization in Primary Care Study, a 5-year study funded by the National Institute of Mental Health (NIMH) and carried out in 3 family practice sites in and around Mobile, Ala. Patients were followed for 24 months after enrollment with assessments of functional status at baseline, 12, and 24 months. After the baseline interview, patients were randomized to 1 of 2 arms of a randomized, single-crossover trial. The primary care physician was notified by a CR letter of the patient's somatization status either immediately following the baseline interview (immediate intervention) or 12 months after enrollment (delayed intervention).

The CR letter that gave recommendations to the primary care physicians for the care of the patients with somatization replicated the letter used in previous studies. ${ }^{18,23-25}$ Key components of the letter include the following:

1. Notification that the patient met criteria for somatization

2. Reassuring information regarding the nonlethal course of somatization

3. Recommendations that the patient be regularly scheduled for brief appointments with the primary care physician and that urgent appointments be avoided as much as possible

4. A recommendation that the physician look closely for signs of disease rather than taking the patient's symptoms at face value

5. A suggestion that hospitalization, surgery, or diagnostic procedures be avoided unless indicated by physical abnormalities

6. A recommendation that the physician view the symptoms as part of an unconscious process rather than telling the patient that the problem is "all in your head"

\section{Screening and Sample Selection}

Practice sites were selected to achieve geographic and socioeconomic diversity, with one urban practice serving a racially and economically diverse population and one suburban and one rural practice, both serving populations with higher socioeconomic levels. In each practice, an interviewer screened patients in full-day blocks distributed evenly throughout the week. Patients were told that the purpose of the study was to investigate symptoms and complaints in primary care 
patients, testing a set of recommendations that would be given to the physicians of those patients with a lot of unexplained symptoms. Patients who presented for care for any reason during the screening days were asked before the visit to complete an 11 -item screen for unexplained physical symptoms. ${ }^{26,27}$ In all, 2,902 consecutive patients were screened. The screen covered a subset of the 35 symptoms from the DSM-III-R criteria for SD. ${ }^{10}$ All patients with 3 or more positive items on the screen were invited to enroll in the study, along with a random sample of those with fewer than 3 symptoms, the latter sample frequency matched to the age/race/sex distribution of the combined somatization study groups. Written informed consent was secured in accordance with the protocol approved by the Institutional Review Board of the institution of the primary research team at the time of the study.

Patients who agreed to participate were scheduled for a face-to-face enrollment interview either in the physician's office or in their own home within 14 days after the index visit. Each enrollee completed the somatization section of the NIMH Diagnostic Interview Schedule (DIS), Version III-R, which measures lifetime as well as current symptoms according to DSM criteria. ${ }^{28}$ On the basis of DIS interview results, enrollment proceeded until there were sufficient patients to fill 3 approximately equal-sized groups:

- SD with 13 or more lifetime unexplained physical symptoms from the somatization section of the DIS,

- Subthreshold somatization with 6 to 12 lifetime unexplained symptoms, and

- Controls with fewer than 6 lifetime unexplained physical symptoms.

The sample was thus originally set up to investigate differences in 3 mutually exclusive groups that represented a continuum in number of unexplained lifetime symptoms.

The analyses reported in this paper required a reclassification of the enrollment interview data to categorize each patient as meeting criteria for SD, ASD, MSD, or no somatization diagnosis, allowing patients to meet criteria for 1 or more of the somatization diagnoses. Patients who did not meet criteria for any somatization diagnosis were not included in any of the analyses reported in this paper. The SD group was not affected by this reclassification. The new ASD group included almost all of the patients from the SD and subthreshold somatization groups. The new MSD group contained a mix of patients from the SD and subthreshold somatization groups. Thus, the new MSD and ASD groups were drawn from the original study groups and contain an oversampling of patients with SD. This resulted in an overrepresentation of patients in the MSD and ASD groups also meeting the more severe SD criteria. To adjust for this in the analyses, design weights based on the ratio of the total number of patients within a screening stratum to the number selected for the study were constructed to reduce the relative influence of the SDs within the ASD and MSD diagnostic groups to more closely approximate our consecutive sample of primary care patients. ${ }^{14}$

\section{Instruments and Measures}

Sociodemographic covariates were collected at baseline and included age, sex, race (minority vs non-minority), education (highest grade completed), and Hollingshead social class (scored from 1 [low] to 5 [high]). ${ }^{29}$ Comorbid psychiatric diagnoses made at baseline using the DIS (DSM-III-R version) included major depression, dysthymia, panic disorder, substance disorder, or posttraumatic stress disorder (PTSD) within the past 12 months. ${ }^{28}$ Comorbid chronic physical diagnoses were measured using a checklist from the Medical Outcomes Study completed by patients at baseline ${ }^{30}$ and reviews of the patients' medical records at baseline, with a positive finding defined by either patient report or medical record report. Chronic physical diseases assessed in this manner and used in these analyses included hypertension, diabetes, cardiac disease (myocardial infarction, enlarged heart, congestive heart failure, or angina), and pulmonary disease (asthma, chronic bronchitis, or emphysema).

Functional status was evaluated using the emotional functioning (MCS) and physical functioning (PCS) subscales of the Short Form-36 (SF-36). ${ }^{31,32}$ Follow-up assessments of functional status were carried out by telephone interviewers unaware of the intervention condition at 12 and 24 months postenrollment, yielding a longitudinal data structure with observations nested within patients over time.

\section{Statistical Analysis}

Statistical analysis was performed using SAS, Version 8 For the Personal Computer. ${ }^{33}$ As preliminary analyses, $\chi^{2}$ tests and $t$-tests were used to assess adequacy of randomization for all 188 patients who met criteria for a somatization diagnosis, comparing patients randomized to immediate intervention with the remaining patients on baseline sociodemographic and clinical characteristics.

Next, exploration of the patterns of missing data over time was carried out to determine the appropriate analytic approach and to identify potential covariates to be included in the analysis. We compared subjects who were observed at 12 and 24 months with subjects missing at these time points with respect to baseline sociodemographic and clinical characteristics and physical and emotional functioning. Likelihood-based methods (general linear mixed models, multilevel mod- 


\begin{tabular}{|c|c|c|c|c|c|c|}
\hline \multirow[b]{3}{*}{$\begin{array}{l}\text { Diagnostic } \\
\text { Classification }\end{array}$} & \multirow{2}{*}{\multicolumn{2}{|c|}{$\begin{array}{c}\text { Abridged Somatization } \\
\text { Disorder }(\mathrm{N}=183) \\
\text { Timing of Intervention }\end{array}$}} & \multirow{2}{*}{\multicolumn{2}{|c|}{$\begin{array}{c}\text { Multisomatoform } \\
\text { Disorder }(\mathrm{N}=111)\end{array}$}} & \multirow{2}{*}{\multicolumn{2}{|c|}{$\begin{array}{c}\text { Somatization } \\
\text { Disorder }(\mathbf{N}=88) \\
\text { Timing of Intervention }\end{array}$}} \\
\hline & & & & & & \\
\hline & $\begin{array}{l}\text { Delayed } \\
(\mathrm{n}=92)\end{array}$ & $\begin{array}{l}\text { Immediate } \\
(\mathrm{n}=91)\end{array}$ & $\begin{array}{l}\text { Delayed } \\
(\mathrm{n}=55)\end{array}$ & $\begin{array}{l}\text { Immediate } \\
(\mathrm{n}=56)\end{array}$ & $\begin{array}{c}\text { Delayed } \\
(\mathrm{n}=43)\end{array}$ & $\begin{array}{c}\text { Immediate } \\
(n=45)\end{array}$ \\
\hline Age, years & $47.5(14.0)$ & $47.6(14.1)$ & $45.4(13.8)$ & $47.7(13.8)$ & $45.5(14.5)$ & $48.2(14.7)$ \\
\hline \multicolumn{7}{|l|}{ Mean (SD) } \\
\hline \% Minority & 20 & 16 & 25 & 16 & 28 & 22 \\
\hline$\%$ Male & 11 & 6 & 7.3 & 3.6 & 5 & 2 \\
\hline Hollingshead social class & $3.6(1.0)$ & $3.4(1.0)$ & $3.4(1.1)$ & $3.3(1.1)$ & $3.2(1.1)$ & $3.2(1.1)$ \\
\hline \multicolumn{7}{|l|}{ Mean (SD) } \\
\hline $\begin{array}{l}\% \text { with any physical } \\
\text { comorbidity }\end{array}$ & 64 & 71 & 60 & 73 & 74 & 73 \\
\hline $\begin{array}{l}\% \text { with any psychiatric } \\
\text { comorbidity }\end{array}$ & 45 & 36 & 49 & 44 & 56 & 49 \\
\hline PCS scores Mean (SD) & $44.3(11.5)$ & $42.4(11.4)$ & $42.4(11.4)$ & $41.3(11.6)$ & $42.2(11.8)$ & $40.3(11.7)$ \\
\hline MCS scores Mean (SD) & $48.5(9.2)$ & $48.9(9.2)$ & $47.6(9.8)$ & $47.8(9.9)$ & $47.5(9.7)$ & $48.7(9.6)$ \\
\hline
\end{tabular}

els, random regression models, hierarchical linear models) using all available data (SAS Proc Mixed) were chosen as the primary analytic approach, since this would minimize the possibility of bias from missing observations and subject dropout that could occur in a complete case analysis such as repeated measures analysis of variance. ${ }^{34-38}$ If "missingness" is determined to be ignorable (ie, "missing completely at random" or "missing at random"), likelihood-based longitudinal methods using all available data along with covariates that explain the missing data mechanism can be used to obtain unbiased estimates of intervention effects. ${ }^{34-39}$ Preliminary examination indicated that an unstructured variance-covariance matrix should be used for all longitudinal analyses.

To test the primary hypothesis that MSD subjects whose physicians received the CR letter would have improved physical and emotional functioning after 12 months compared with subjects receiving usual care, longitudinal analyses were carried out to model patient responses over time. ${ }^{34,37,38,40-42}$ We used repeated measures rather than growth curve modeling because of the crossover design, where time is fixed and categorical. When the global test of the intervention effect over time was significant, specific linear contrasts were used to estimate the 12-month intervention effect, defined as the 12 -month change in the intervention group minus the 12 -month change in the usual care group, after adjusting for baseline covariates. ${ }^{34,43}$

Since the somatization diagnostic groups overlapped, separate analyses were performed to assess intervention effects on functional status for subjects meeting criteria for ASD and for SD alone. Within each diagnostic category, subjects randomized to immediate intervention were compared with subjects in the delayed intervention group using longitudinal analysis, as described above.

For MSD patients, to determine whether the CR letter intervention had differential effects on physical or emotional functioning by patient characteristics, interaction effects between intervention group and the covariates listed above over time were investigated.

Sensitivity analysis using multiple imputation and pattern mixture models to handle missing data were carried out and yielded similar results in all analyses. Therefore, reports of longitudinal analyses based on all available data are included here. Additionally, sensitivity analyses were carried out comparing weighted to unweighted analyses. Since results of the unweighted analyses were very similar, only weighted analyses are reported here.

Finally, in order to decide whether changes in health-related quality of life (HRQOL) were meaningful, we calculated a criterion based on the standard error of measurement (SEM) for identifying meaningful changes in HRQOL using baseline standard deviation and reliability for PCS and MCS. ${ }^{44-46}$ We estimated the average reliability (internal consistency) for the SF-36 summary scales to be 0.86 in our sample, which agrees closely with the reported reliabilities for the MCS and PCS in US populations. ${ }^{47}$ The SEM, used as an estimate of minimal clinically important difference, ${ }^{45}$ was computed as the standard deviation of the score multiplied by the square root of 1 minus its reliability coefficient. ${ }^{46}$ 


\section{RESULTS}

Sociodemographic and clinical characteristics of the patients are presented in Table 1, organized by diagnosis and intervention group. Altogether, 188 patients met criteria for 1 or more somatization diagnoses: 183 of these patients met criteria for ASD, 111 for MSD, and 88 for SD. Of interest, while $28.8 \%$ of primary care patients with MSD met criteria for 1 -year major depression/dysthymia, $55.2 \%$ of primary care patients with 1 -year major depression/dysthymia met criteria for MSD. Further details on the results of the screening and recruitment process, the prevalence of SD, MSD, and ASD, and the extent of overlap of MSD with SD and ASD are presented in a previous paper. ${ }^{14}$ There were no significant differences between patients assigned to the intervention groups and usual care at baseline with respect to age, race, sex, social class, physical comorbidity, psychiatric comorbidity, or physical or emotional functioning for the total sample of somatizing patients or within any of the 3 diagnostic categories (Table 1). Follow-up rates were $80.5 \%$ at 12 months and $60.5 \%$ at 24 months. Analysis of missing versus nonmissing subjects, performed at 12 and 24 months for the entire sample of 188 somatizers, indicated no significant differences on baseline sociodemographic or clinical covariates (Table 2). Although there was a tendency for 24-month dropouts to have slightly lower baseline MCS scores, the assumption of "missing at random" (ignorable missingness) was not violated.

In the longitudinal analysis of PCS scores for MSD

subjects, adjusting for baseline sociodemographic and clinical covariates and baseline MCS scores as described in the Methods section, the global test for differences between the immediate and delayed intervention groups over time was significant $(P=.0002)$. On average, the immediate intervention group maintained physical functioning at baseline levels during the intervention year while the delayed intervention group that received usual care during this year actually declined in physical functioning. The 12 -month intervention effect on PCS scores, assessed using change scores as described in the Methods section, was 5.5 points (95\% CI, 2.5 to 8.4 ; effect size, 0.48), compared with the estimated minimal clinically important difference of 4.7 points. Since the study design was a randomized controlled trial, we also estimated the 12 -month intervention effect in a model without any covariates (eg, based on crude means for the groups over time). The intervention effect of 5.6 points was statistically significant $(P<.001)$ and very close to that obtained from the full model with covariates. A crossover effect was observed in both models during year 2 , with substantial improvement in the delayed intervention patients from 12 to 24 months. Thus, the patients in the delayed intervention group, who had demonstrated a decline in functioning between baseline and 12 months, returned to baseline levels of physical functioning after they received the intervention at 12 months. Meanwhile, patients whose providers received the CR letter at baseline demonstrated a decline in functioning from 12 to 24 months (Figure 1).

In a separate analysis in

\section{Table 2. Differences Between Missing and Present Subjects at 12 and 24 Months}

\begin{tabular}{|c|c|c|c|c|}
\hline \multirow[b]{2}{*}{ Status } & \multicolumn{2}{|c|}{12 Months } & \multicolumn{2}{|c|}{24 Months } \\
\hline & $\begin{array}{l}\text { Missing } \\
(n=37)\end{array}$ & $\begin{array}{c}\text { Present } \\
(n=151)\end{array}$ & $\begin{array}{l}\text { Missing } \\
(n=74)\end{array}$ & $\begin{array}{c}\text { Present } \\
(n=114)\end{array}$ \\
\hline Age, years & $48.5(14.0)$ & $47.1(13.9)$ & $45.5(14.2)$ & $48.6(1.3)$ \\
\hline \multicolumn{5}{|l|}{ Mean (SD) } \\
\hline \% Minority & 19 & 19 & 18 & 20 \\
\hline$\%$ Male & 8 & 7 & 8 & 7 \\
\hline Hollingshead social class & $3.6(1.0)$ & $3.5(1.0)$ & $3.4(1.0)$ & $3.5(1.0)$ \\
\hline \multicolumn{5}{|l|}{ Mean (SD) } \\
\hline $\begin{array}{l}\% \text { with physical } \\
\text { comorbidity }\end{array}$ & 73 & 66 & 66 & 68 \\
\hline $\begin{array}{l}\% \text { with psychiatric } \\
\text { comorbidity }\end{array}$ & 49 & 38 & 43 & 39 \\
\hline Baseline PCS scores & $44.5(12.4)$ & $43.0(11.3)$ & $44.9(11.6)$ & $42.9(11.5)$ \\
\hline \multicolumn{5}{|l|}{ Mean (SD) } \\
\hline Baseline MCS scores* & $48.2(10.0)$ & $48.9(9.0)$ & $47.0 *(10.2)$ & $49.9 *(8.3)$ \\
\hline \multicolumn{5}{|l|}{ Mean (SD) } \\
\hline$\%$ year 1 intervention & 41 & 50 & 45 & 51 \\
\hline \multicolumn{5}{|c|}{$\begin{array}{l}t \text {-Test and } \chi^{2} \text { tests used to compare missing to nonmissing } \\
{ }^{*} \text { Baseline MCS scores for } 24 \text { month missing vs nonmissing, } P=.04 \text {; all other } P \text { values }>.10 \\
S D=\text { standard deviation; PCS = physicial functioning and MCS = emotional functioning subscales of Short Form-36 }\end{array}$} \\
\hline
\end{tabular}

patients meeting criteria for $\mathrm{SD}$, we estimated the 12 month intervention effect on PCS scores to be 7.1 points, after adjusting for sociodemographic and clinical covariates and baseline MCS scores $(95 \% \mathrm{CI}, 3.5$ to 10.7 ; effect size, 0.61). In another analysis of patients meeting criteria for ASD using the same covariates as above, we estimated the 12-month intervention effect for ASD to be 4.2 points ( $95 \% \mathrm{CI}$, 1.5 to 6.9 ; effect size, 0.37 ).

The intervention did not significantly impact mental health functioning at either 12 or 24 months in the 3 separate analyses that included subjects meeting criteria for 


\section{Figure 1. Physical Functioning Scores by Intervention Group for Multisomatoform Disorder Subjects.}

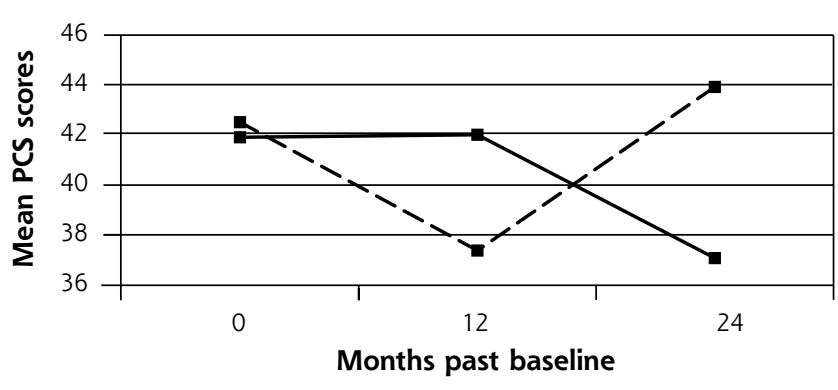

$\longrightarrow$ Immediate intervention $\quad-\rightarrow-$ Delayed intervention mographic diversity. Previous studies of the CR letter intervention used patients singled out by primary care physicians for study; such severely affected patients were likely to respond differently to an intervention than a representative primary care population. This study also provides the first investigation of CR-letter impact in patients with MSD. Multisomatoform disorder is highly prevalent and entails serious impairment. ${ }^{13,14}$ The diagnosis of MSD is easier to make and more reliable than other somatization diagnoses, since it is based on a shorter list of symptoms and since it involves only current symptoms. We believe that these results suggest that MSD is a useful diagnostic classification for studying and managing somatization in primary care patients.

The pattern of the effects in the 2 intervention groups is of interest. The impact of the intervention on the immediate intervention group during the first 12 months was primarily seen in the preservation of physical functional health when compared with a striking decline of physical functioning in the delayed intervention group. However, the delayed intervention group, which received the intervention at 12 months, displayed an improvement of functional health back to the original baseline level between 12 and 24 months. Thus, the intervention either maintained functional health at baseline levels (in the immediate intervention group) or brought functioning back to original baseline levels (in the delayed intervention group). The apparent difference in the immediate postintervention change (or slope) in the 2 groups is largely attributable to the decline in functioning in the delayed intervention group during the 12 months of usual care. The decline in functional health seen in the immediate intervention group during the 12 - to-24-month period after the intervention suggests that there may be a need for a follow-up "booster" letter or some other system for reminding the physicians of the diagnosis and the treatment recommendations.

The mechanism of action of the CR-letter intervention that produces this impact on physical functioning is not well understood. Previous authors have hypothesized that the CR letter may prompt physicians to refrain from ordering invasive procedures in the pursuit of patient complaints, preventing physical impairment related to these procedures and reducing patient fears regarding the possible presence of a serious physical condition. ${ }^{24}$ The intervention's stronger effect in the presence of comorbid chronic physical disease provides additional information that may help in further understanding how the letter intervention effects a change in 
the patient's condition. An important part of primary care for patients with chronic physical disease involves educating and monitoring them for the development of symptoms that may indicate complications of the disease or its treatment. For somatizing patients, this monitoring may inadvertently increase patient vigilance about physical sensations that might otherwise be ignored. When symptoms arise in this context, physicians generally evaluate the patient aggressively, even in the absence of conclusive findings. Such investigation tends to increase patient anxiety, hypervigilance, and impairment. In this context, we suspect that an "active ingredient" of the CR letter is the provision of the somatization diagnosis, which reduces clinical uncertainty. While physical symptoms suggestive of complications cannot be ignored, the CR letter encourages the primary care physician to accept a reasonable level of normal findings as evidence that complications of the patient's chronic disease are not developing. Further exploration of the impact of patient factors on the effect of the intervention might help in determining whether chronic physical disease is itself the element discriminating patients who do and do not respond to the CR letter or whether it is a proxy for an underlying construct.

The study has several limitations. Our screening methodology was not efficient for men, as described in more detail in a previous paper. ${ }^{14}$ This method produced a small but representative sample of men available for analysis. Also, this relatively large sample of primary care patients drawn from 3 diverse practice settings comes from 1 geographic region of the United States, where patterns of somatization may be different from other regions. ${ }^{48}$ Additionally, the effect of the CR letter in subjects meeting criteria for the 3 somatization classifications could not be directly compared statistically, and our findings may overstate the similarities and understate the differences among the diagnoses because of the overlap in patients meeting criteria for the 3 diagnoses. A study using 3 mutually exclusive groups of patients would be better for this purpose. We also recognize that we did not evaluate how this intervention affected expenditures, although previous studies have reported cost savings in fee-for-service settings. ${ }^{18,23-25}$ Further research is needed to evaluate the effect of the CR letter on health care costs in capitated plans. Finally, while the power to detect a medium effect size (0.5) based on differences at 12 months between MSD immediate and delayed intervention groups is $72 \%$, there was not sufficient power to detect all of the factors that might contribute to a differential response to the CR letter intervention. It is possible that patient or physician features other than chronic physical disease might have an impact on the response to the intervention.
Somatization imposes a tremendous burden on both patients and the health care system. It is imperative that we develop and refine effective interventions for somatization that can be broadly implemented in primary care populations. This study provides evidence that a CR letter intervention has an impressive impact on physical impairment of a consecutive sample of primary care patients with multisomatoform disorder. This intervention works especially well for patients with comorbid chronic physical disease. Further research is warranted to refine the CR letter intervention and to design alternative interventions for patients who do not benefit from the CR letter.

To read commentaries or to post a response to this article, see the online version at http://www.annfammed.org/cgi/content/full/1/4/228.

Funding support: This work was supported in part by MH45441 and MH63651 from the National Institute of Mental Health and PE84054 from the Bureau of Health Professions, Bethesda, Md.

Key words: Somatoform disorders; mental disorders; primary care

Received August 7, 2002; received, revised, October 18, 2002; accepted November 11, 2002.

\section{References}

1. Kroenke K, Mangelsdorff D. Common symptoms in ambulatory care: incidence, evaluation, therapy, and outcome. Am J Med. 1989;86: 262-266.

2. Barsky AJ, Borus JF. Somatization and medicalization in the era of managed care. JAMA. 1995;274:1931-1934.

3. Kroenke K. Symptoms in medical patients: an untended field. Am J Med. 1992;92:3S-6S.

4. Swartz M, Landerman R, George LK, Blazer DG, Anthony JC. Somatization disorder. In: Robins LN, Regier DA, eds. Psychiatric Disorders in America: The Epidemiologic Catchment Area Study. New York, NY: The Free Press; 1991.

5. Shaw J, Creed F. The cost of somatization. J Psychosom Res. 1991;35: 307-312.

6. Escobar JI, Golding JM, Hough RL, Karno M, Burnam MA, Wells KB. Somatization in the community: relationship to disability and use of services. Am J Public Health. 1987;77:837-840.

7. Lin EH, Katon W, Korff MV, et al. Frustrating patients: physician and patient perspectives among distressed high utilizers of medical services. J Gen Intern Med. 1991;6:241-246.

8. Hahn SR, Kroenke K, Spitzer RL, et al. The difficult patient: prevalence, psychopathology, and functional impairment. J Gen Intern Med. 1996;11:1-8.

9. Hahn SR, Thompson KS, Wills TA, Stern V, Budner NS. The difficult doctor-patient relationship: somatization, personality and psychopathology. J Clin Epidemiol. 1994;47:647-657.

10. American Psychiatric Association. Diagnostic and Statistical Manual of Mental Disorders. $3^{\text {rd }}$ ed. Washington, DC: American Psychiatric Association; 1987.

11. Escobar J, Burnam M, Karno M, Forsythe A, Goulding J. Somatization in the community. Arch Gen Psychiatry. 1987;44:713-718.

12. Escobar JI, Rubio-Stipec M, Canino G, Karno M. Somatic symptom index (SSI): a new and abridged somatization construct. Prevalence and epidemiological correlates in two large community samples. J Nerv Ment Dis. 1989; 177:140-146. 
13. Kroenke K, Spitzer RL, deGruy FV, et al. Multisomatoform disorder: an alternative to undifferentiated somatoform disorder for the somatizing patient in primary care. Arch Gen Psychiatry. 1997;54:352-358.

14. Dickinson W/P, Dickinson LM, deGruy FV, et al. The somatization in primary care study: a tale of three diagnoses. Gen Hosp Psychiatry. 2002;25:1-7

15. Simon G, Gureje O. Stability of somatization disorder and somatization symptoms among primary care patients. Arch Gen Psychiatry. 1999;56:90-95.

16. Escobar Jl. Pharmacological treatment of somatization/hypochondriasis. Psychopharmacol Bull. 1996;32:589-596.

17. Menza M, Lauritano M, Allen $\mathrm{L}$, et al. Treatment of somatization disorder with nefazodone: a prospective, open-label study. Ann Clin Psychiatry. 2001;13:153-158.

18. Kashner TM, Rost K, Smith GR, Lewis S. An analysis of panel data. The impact of a psychiatric consultation letter on the expenditures and outcomes of care for patients with somatization disorder. Med Care. 1992:30:811-821.

19. Kroenke K, Swindle R. Cognitive-behavioral therapy for somatization and symptom syndromes: a critical review of controlled clinical trials. Psychother Psychosom. 2000;69:205-215.

20. Cherkin D, Eisenberg D, Sherman K, et al. Randomized trial comparing traditional Chinese medical acupuncture, therapeutic massage and self-care education for chronic low back pain. Arch Intern Med. 2001; 161:1081-1088.

21. Field TM, Sunshine W, Hernandez-Reif M, et al. Massage therapy effects on depression and somatic symptoms in chronic fatigue syndrome. J Chronic Fatigue Syndr. 1997;3(3):43-51.

22. Preyde M. Effectiveness of massage therapy for subacute low-back pain: a randomized controlled trial. Can Med Assoc J. 2000;162: 1815-1820.

23. Smith G Jr, Monson RA, Ray DC. Psychiatric consultation in somatization disorder. A randomized controlled study. N Engl J Med. 1986; 314:1407-1413.

24. Rost K, Kashner TM, Smith GR. Effectiveness of psychiatric intervention with somatization disorder patients: improved outcomes at reduced costs. Gen Hosp Psychiatry. 1994;16:381-387.

25. Smith GR, Rost K, Kashner TM. A trial of the effect of a standardized psychiatric consultation on health outcomes and costs in somatizing patients. Arch Gen Psychiatry. 1995;52:238-243.

26. Smith GJ, Brown FW. Screening indexes in DSM-III-R somatization disorder. Gen Hosp Psychiatry. 1990;12(3):148-152.

27. Swartz M, Hughes D, George L, Blazer D, Landerman R, Bucholz K. Developing a screening index for community studies of somatization disorder. J Psychiatric Res. 1986;20(4):335-343.

28. Robins LN, Helzer JE, Croughan J, Ratcliff KS. National Institute of Mental Health Diagnostic Interview Schedule. Arch Gen Psychiatry. 1981;38:381-389.

29. Hollingshead AB. Four-factor index of social status. New Haven, Conn: Yale University, Department of Sociology; 1975.

30. Ware JE Jr, Brooks RH, Davies-Avery A. Conceptualization and measurement of health for adults in the Health Insurance Study, Vol. 1. In: Corporation R, ed. Model and Health and Methodology. Santa Monica, Calif: Rand Corp; 1980.
31. McHorney C, Ware J, Lu J, Sherbourne C. The MOS 36-Item ShortForm Health Survey (SF-36): III. Tests of data quality, scaling assumptions, and reliability across diverse patient groups. Med Care. 1994; 32:40-66.

32. Ware JE, Snow KK, Kosinski M, Gandek B. SF-36 Health Survey: Manual and Interpretation Guide. Lincoln, RI: Quality Metric Inc; 1993, 2000.

33. SAS: Version 8 for the Personal Computer. Cary, NC: SAS Institute; 1999.

34. Fairclough DL. Design and Analysis of Quality of Life Studies in Clinical Trials. Boca Raton, Fla: Chapman and Hall/CRC Press; 2002.

35. Demster A, Laird N, Rubin D. Maximum likelihood estimation from incomplete data via the EM algorithm. J Royal Stat Soc. 1977. 39:1-38.

36. Diggle $\mathrm{P}$, Kenward $\mathrm{M}$. Informative dropout in longitudinal data analysis. Appl Stat. 1994;43:49-93.

37. Gibbons R, Hedeker D, Elkin I, et al. Some conceptual and statistical issues in analysis of longitudinal psychiatric data. Application to the NIMH treatment of Depression Collaborative Research Program dataset. Arch Gen Psychiatry. 1993;50:739-750.

38. Little R, Rubin D. Statistical Analysis with Missing Data. New York, NY: John Wiley E Sons; 1987.

39. Hedeker D. An introduction to growth modeling. In: Kaplan D, ed Handbook of Quantitative Methodology for the Social Sciences. Thousand Oaks, Calif: Sage Publications; 2002.

40. Raudenbush S, Bryk A. Hierarchical Linear Models: Applications and Data Analysis Methods. 2nd ed. Thousand Oaks, Calif: Sage Publications; 2002.

41. Smith J, Rost K, Nutting P, Elliott C, Dickinson L. Impact of ongoing primary care intervention on long term outcomes in uninsured and insured patients with depression. Med Care. 2002;40:1210-1222.

42. Rost K, Nutting P, Smith J, Elliott C, Dickinson L. Managing depression as a chronic disease: a randomized trial of ongoing primary care depression treatment. BMJ. 2002;325:934-937.

43. Rosner B. Fundamentals of Biostatistics. 5th ed. Pacific Grove, Calif: Duxbury; 2000:309-312.

44. Wyrwich K, Nienaber N, Tierney W, Wolinsky F. Linking clinical relevance and statistical significance in evaluation intra-individual changes in health related quality of life. Med Care. 1999;3:469-478.

45. Wyrwich K, Tierney W, Wolinsky F. Further evidence supporting an SEM-based criterion for identifying meaningful intra-individual changes in health-related quality of life. J Clin Epidemiol. 1999;52: 861-873.

46. Anastasi A, Urbina S. Psychological Testing. 7th ed. Upper Saddle River, NJ: Prentice-Hall; 1997

47. Ware J, Kosinski M, Keller S. SF-36 Physical and Mental Health S ummary Scores: A Users Manual. Boston, Mass: Health Assessment Lab; 1994.

48. Swartz M, Landerman R, Blazer D, George L. Somatization symptoms in the community: a rural/urban comparison. Psychosomatics. 1989; 30:44-53. 